\title{
Characterization of benign thyroid nodules with HyperSPACE (Hyper Spectral Analysis for Characterization in Echography) before and after percutaneous laser ablation: a pilot study
}

\author{
Simona Granchi, Enrico Vannacci, Elena Biagi
}

Department of Information Engineering (DINFO), University of Florence, Florence, Italy

\begin{abstract}
Aims: To evaluate the capability of the HyperSPACE (Hyper SPectral Analysis for Characterization in Echography) method in tissue characterization, in order to provide information for the laser treatment of benign thyroid nodules in respect of conventional B-mode images and elastography. Material and methods: The method, based on the spectral analysis of the raw radiofrequency ultrasonic signal, was applied to characterize the nodule before and after laser treatment. Thirty patients ( 25 females and 5 males, age between 37 and 81 years) with thyroid benign nodule at cytology (Thyr 2) were evaluated by conventional ultrasonography, elastography, and HyperSPACE, before and after laser ablation. Results: The images processed by HyperSPACE exhibit different color distributions that are referred to different tissue features. By calculating the percentages of the color coverages, the analysed nodules were subdivided into 3 groups. Each nodule belonging to the same group experienced, on average, similar necrosis extension. The nodules exhibit different Configurations (colors) distributions that could be indicative of the response of nodular tissue to the laser treatment Conclusions: HyperSPACEcan characterize benign nodules by providing additional information in respect of conventional ultrasound and elastography which is useful for support in the laser treatment of nodules in order to increase the probability of success.
\end{abstract}

Keywords: ultrasonography; signal processing; cluster analysis; laser therapy; thyroid nodule.

\section{Introduction}

Benign thyroid nodules are common in the general population especially in iodine-deficient areas. The use of ultrasound (US) in clinical practice has increased the majority of asymptomatic, benign thyroid nodules which are stable in size and can be monitored with clinical and US examinations [1]. Considering that in large thyroid nodules no effective medical therapies have been identified to reduce their size, surgery, which still represents the common treatment in cold thyroid nodules, may cause local symptoms and patient concern. Recently,

Received 30.09.2016 Accepted 25.02.2017

Med Ultrason

2017, Vol. 19, No 2, 172-178

Corresponding author: Simona Granchi, Ph.D, Research fellow Department of Information Engineering (DINFO), University of Florence, Via S. Marta 3, 50139 Florence, Italy Phone: +39 0552758607, Fax: +390552758570

Email: simona.granchi@unifi.it minimally invasive techniques have been proposed for treating large benign thyroid nodules. Percutaneous ethanol injections are now acknowledged as a rapid, effective, and inexpensive treatment for cystic thyroid lesions [2] whereas thermal ablation with laser or radiofrequency is used more frequently in solid or complex nodules [3]. Other techniques such as cryoablation, highfocused ultrasound, microwaves, and electroporation are not recommended in clinical practice [4]. Over the last few years, several studies [5-11] have demonstrated that percutaneous laser ablation under ultrasound guidance is able to reach a target lesion within the thyroid with great precision. Laser treatment is quick (5-20 minutes) and destroys thyroid tissue in a predictable, repeatable way without any major complications when performed by experienced operators. However, the success of the treatment depends on its monitoring in order to evaluate tissue damage, the margins of the necrotic areas, and the evolution of the results during follow-up. Several studies [12-13] have shown that laser treatment is more effective 
than L-T4 treatment in reducing the nodular volume and related compressive symptoms; therefore, the method seems to be a promising procedure. Conventional US provides information on ultrasound patterns correlated with thyroid pathologies [11,14-16], but the sensitivity, specificity, and accuracy are extremely variable from study to study. As reported in the consensus on thyroid nodules, a firm or hard consistency at palpation is associated with an increased risk of malignancy. However, this clinical parameter is highly subjective and depends on the examiner's experience.

Elastography is a newly developed dynamic technique that uses US to give an estimation of tissue stiffness. In addition, US can be used to obtain information on the composition of the nodular tissue by using spectral analysis of the radiofrequency signal (RF) which contains information about ultrasound-tissue interactions [16-19]. Through the identification of new coefficients [20-26], the spectral analysis of the RF signal is able to provide information regarding the biological structure of the tissue. The use of RF could therefore be an interesting support for conventional US as it provides information about the condition of the tissue and the modifications occurring during and after laser ablation, which can thus be use dto establish its effectiveness.

The proposed investigation method for spectral analysis called HyperSPACE (Hyper SPectral Analysis for Characterization in Echography) [27-30] implements a sub-band decomposition of the local signal spectrum and operates in hyperspectral domain. Important results of this method were obtained from an experiment involving ten Italian hospital clinics conducted to differentiate the two most common breast pathologies [27-30].

In this research, HyperSPACE was applied to characterize the structure of benign nodular thyroid tissue before laser treatment and the results obtained were compared with those of conventional US and elastography and correlated with the extension of the necrosis area induced by laser ablation.

\section{Materials and methods}

\section{Investigation Method}

The method is based on the spectral analysis of the RF ultrasonic signal and consists of several steps fully described in previous published papers [27-30].

In the first step, the RF signal is filtered by means of a bank of $\mathrm{N}$ filters, performing an $\mathrm{N}$ sub-bands decomposition of the spectral band. In the second step, the coefficients obtained from the decomposition are analyzed and processed in $\mathrm{N}$ dimensional spectral hyperspace in order to generate the HyperSPACE coefficients (hs) [30] that are subjected to a training phase. In the training phase, the $h s$ coefficients, belonging to selected Regions of Interest (ROIs) on the B-mode image, are trained to identify sets of spectral parameters, called Configurations, able to characterize the investigated ROIs. In particular, during this phase, the physician selects the area of the thyroid nodule on the B-mode, which is automatically subdivided into three different ROIs by the algorithm as shown on the left of figla. On the right of the same figure, the $h s$ distributions of red, green, and blue ROIs, are represented in a three-dimensional projection of hyperspace and it is interesting to note that the coefficients have different shapes and positions for each ROI in the hyperspace considered. In the fourth step, called Classification, the algorithm detects the presence of the previously defined Configurations over the entire RF image. By processing $h s$ coefficients [30], several clusters relating to each ROI are obtained (fig 1b) and the hyper volume that contains the combination of all clusters, relating to each ROI, is called Configuration (fig 1c).

During the Classification phase, Configurations detected over the entire image are visualized on the Bmode image by superimposing a color code associated with each Configuration, obtaining the HyperSPACE image. Therefore, if, for example, three Configurations are present in a RF image, the final B-mode will have three-
B - mode

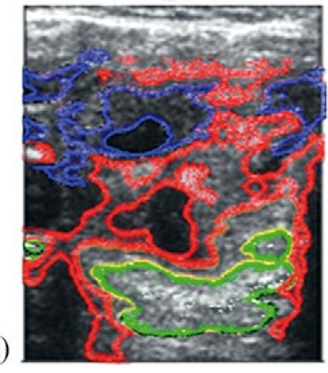

Clusters of hs coefficients

b)

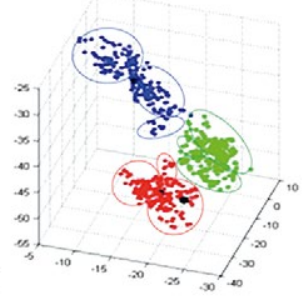

3D hyperspace

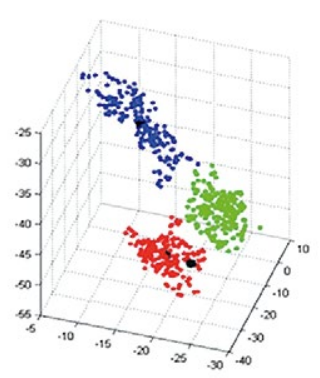

Final Configurations

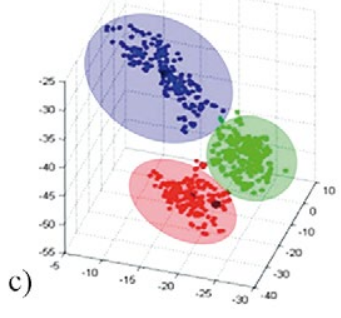

Fig 1. Training phase: a) on the left, the B mode with automatic selected ROIs. On the right, the 3D hyperspace with hs coefficients related to the selected ROIs; b) Clusters of hs coefficients; c) Final Configurations which are N-dimensional volumes containing the clusters inside. 


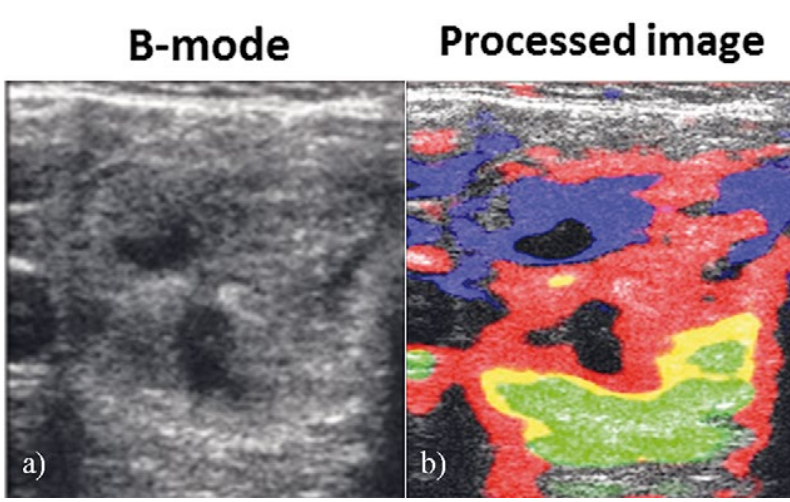

Fig 2. Classification phase: a) B-mode representation; b) HyperSPACE Image: result of the superimposition of a color code on the gray-scale B-Mode. During this phase, the detection of the identified Configurations is applied on the entire RF frame.

color codes corresponding to the areas identified by these three Configurations, as shown in fig 2.

\section{The Pilot Study}

The Hyper-SPACE was applied on data acquired at the Endocrinology 1 Department of the University Hospital of Pisa (Italy) [31-35]. In this study, all patients undergoing clinical investigation signed an informed consent form that allowed them to participate. The Ethics Committee approved the clinical protocol, which defined the inclusion and exclusion criteria for enrolling patients, the characteristics of the acquisition procedure, the diagnostic tests to be performed on the patients, and the type of data to be collected. The inclusion criteria established that all patients had to be 20 years of age or older, with the presence of a single or dominant nodule, well defined both clinically and with ultrasound. The nodular volume was $8-24 \mathrm{ml}$ (maximum diameter of $3-5 \mathrm{~cm}$ ). At the scintiscan, the nodule had to be cold. All patients were euthyroid with undetectable serum calcitonin and all nodules were benign at cytology (Thyr 2).

The set up used for the acquisition of RF echographic signal consisted of the ultrasound equipment Mylab 70 (Esaote S.p.A, Florence, Italy), optically connected to a FEMMINA [36] a versatile system that makes it possible to acquire and process the RF signal in real time. The probe used was the linear array LA435 (Esaote S.p.A, Florence, Italy) with a nominal frequency of $10 \mathrm{MHz}$. The instrumental acquisition parameters were: excitation frequency $12.5 \mathrm{MHz}$, Power $80 \%$ of dynamic, TGC $50 \%$ of dynamic, at every depth, total gain $65 \%$ of dynamic, and focus depth on nodule.

Conventional US was performed before laser treatment. During the US examination, 20 RF frames were acquired for each nodule section (sagittal and transversal) from each patient. Laser Ablation Therapy (LAT) was carried out in a single session by inserting two 21-gauge spinal needles into the target thyroid lesion under US monitoring. The ablation was performed with a 1064$\mathrm{nm}$ neodymium yttrium-aluminum garnet (Nd-YAG) laser (Echolaser; Elesta, Italy) with an output power of $3 \mathrm{~W}$. Energy delivery was $3600 \mathrm{~J}$ for nodules $6-13 \mathrm{~mL}$ and $7200 \mathrm{~J}$ for nodules measuring $14-17 \mathrm{ml}$. With US monitoring, the area under treatment was visualized as a hyperechoic zone that gradually enlarged due to the formation of gas micro-bubbles in the coagulated tissue. After an interval of 2-3 hours following laser treatment, the patient was examined again with the echographic equipment and additional RF frames of nodules were acquired as per the previous procedure. In this work, in order to characterize the nodular tissue before treatment, $1200 \mathrm{RF}$ frames deriving from 30 patients were processed. The data set of enrolled patients was composed of 25 females and 5 males aged between 37 and 81 years [mean age \pm standard deviation (SD) $53 \pm 12$ ].The training set consisted of $24 \mathrm{RF}$ frames relating to 8 patients, randomly selected from transversal and sagittal sections. All the other frames were used in the classification. In this study, only three Configurations represented by means of the colors Red (R), Green (G) and Blue (B) were chosen to be developed. Moreover, by calculating the coverage percentage of each color in respect of nodular volume and the reciprocal ratios of these percentages in the HyperSPACE images, the nodules were divided into three different groups (A, B, and C), defined by means of the clustering algorithm k-means [37]. After that, every nodule belonging to each group was correlated to the percentage of necrosis extension, in order to correlate the results with the damage inflicted by laser ablation.

\section{Results}

The RF images related to the 30 patients, described above, were processed through the algorithm and, for each patient, the percentages of colored areas in the HyperSPACE images before ablation was estimated obtaining the distribution reported in the histogram of fig 3 .

The three groups, wherein the nodules were subdivided, exhibit mean values of the colors coverage that are different from each other, as reported in figure 4 and in Table I.

Examples of HyperSPACE images for each group are represented in fig 5.

In order to verify if different colors distributions and, accordingly, if different tissue features of nodules were related to different responses to laser treatment, the ratios between necrosis volume (VNEC) and nodule volume (VNOD) were calculated for all nodules obtaining the 


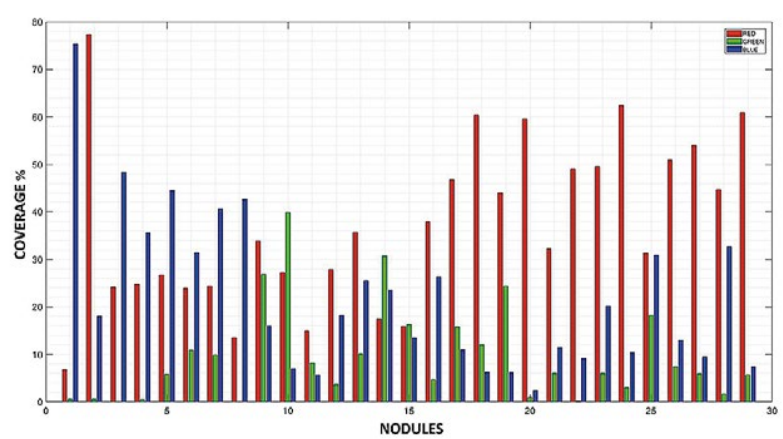

Fig 3. Histogram of red (R), green (G), and blue (B) colors percentages distribution for all 30 patients

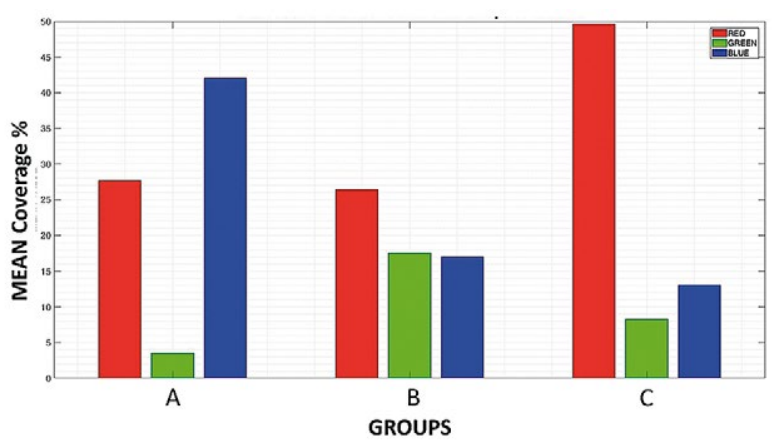

Fig 4. Histogram of the mean values of the red, green, and blue colors percentage for each group (A, B, C).
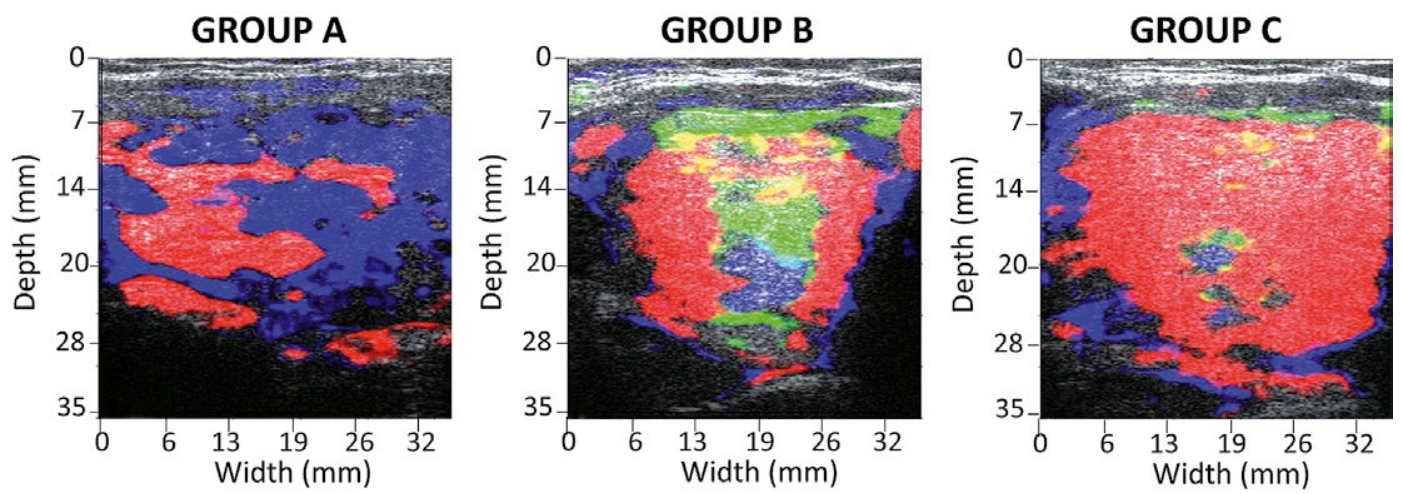

Fig 5. Examples of HyperSPACE images for each group. The colors distributions in the HyperSPACE images are different and this may be due to the different composition and structural organization of the analyzed nodules.

Table I. Coverage percentage of colors red, green, blue for each group.

\begin{tabular}{llllllllll}
\hline GROUP & $\mathbf{R \%}$ & \multicolumn{3}{c}{$\mathbf{G \%}$} & \multicolumn{3}{c}{ B\% } \\
\cline { 2 - 11 } & Mean & SD & Median & Mean & SD & Median & Mean & SD & Median \\
\hline A & 27.7 & 21.2 & 24.3 & 3.5 & 4.6 & 0.6 & 42.1 & 16.4 & 41.7 \\
B & 26.4 & 9.3 & 27.6 & 17.5 & 13.4 & 13.2 & 17.0 & 7.9 & 17.1 \\
C & 49.7 & 10.1 & 49.5 & 8.3 & 7.3 & 6.0 & 13.1 & 9.3 & 10.4 \\
\hline
\end{tabular}

$\mathrm{R} \%$ : coverage percentage of red, G\%: coverage percentage of green, $\mathrm{B} \%$ : coverage percentage of blue, SD: standard deviation.

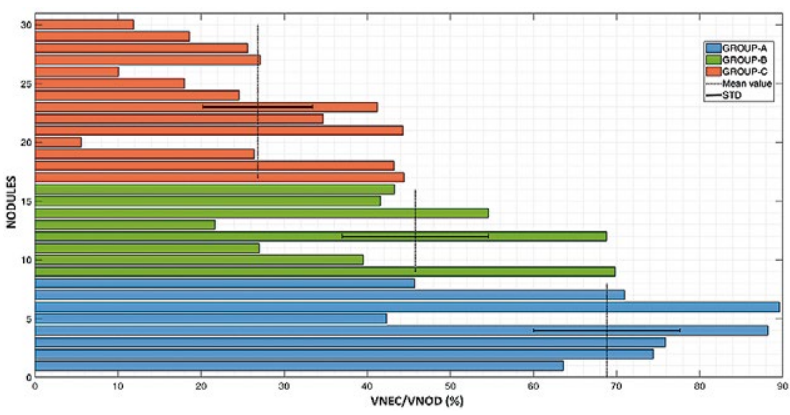

Fig 6. Representation of the distribution of ratios between the necrosis volume (VNEC) and nodule volume (VNOD) for all 30 patients, divided in the three group A, B, and C. For each group the mean values of VNEC/VNOD is displayed through the black dashed vertical line, with the standard deviation (SD) represented by means of the red solid horizontal segment line. histogram shown in fig 6, where values are represented taking into account the group to which they belong. For each group the mean value, standard deviation and median were estimated, as reported in Table II. The extensions of necrosis were estimated by measuring the nonperfused area in the B-mode image of the ablated nodule after injection of the contrast agent.

Moreover, a comparison of HyperSPACE and elastosonography images was performed with the aim to put in evidence the different information coming from one or the other. In fig 7 it can be observed that all three nodules have similar elastosonography images, (their score is equal to one in each case). On the other hand, their HyperSPACE images are different and each nodule belongs to a different group with a different range of necrosis 
Table II. Ratio between the necrosis volume induced by ablation and the nodular volume before ablation for each group.

\begin{tabular}{llll}
\hline GROUP & \multicolumn{3}{l}{ VNEC/ } \\
\cline { 2 - 4 } & Mean & SD & Median \\
\hline A & 68.8 & 17.6 & 72.7 \\
B & 45.8 & 17.6 & 42.4 \\
C & 26.8 & 13.2 & 26.0 \\
\hline
\end{tabular}

VNEC: necrosis volume, VNOD: nodule volume, SD: standard deviation

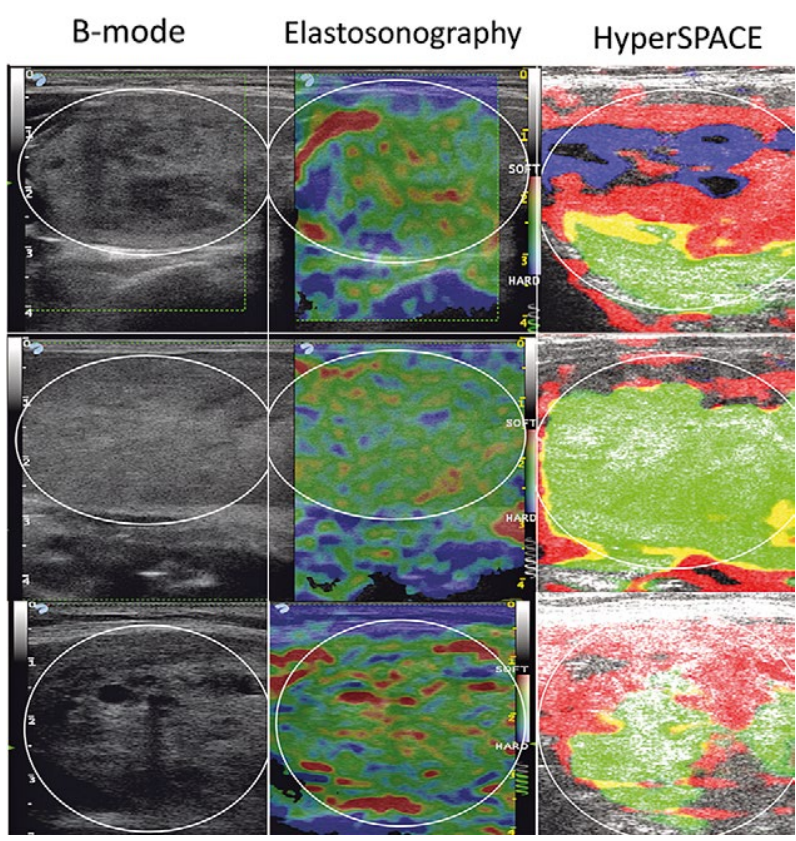

Fig 7. Three examples of benign nodules. The first column contains the B-mode representation. In the middle, the elastosonography images are shown and the right-hand column contains the HyperSPACE images. The three nodules have similar elastosonography images $($ score $=1)$, while the HyperSPACE images exhibit different color distribution.

extension. In addition, areas with the same echographic appearance in traditional B-mode are characterized by different Configurations.

\section{Discussions}

Percutaneous laser ablation is considered a minimally invasive surgical technique that offers numerous benefits for the patient and excellent results in reducing thyroid nodules. The effectiveness of this type of technique may be increased by using a monitoring system capable of evaluating not only the damage induced in the tissue during treatment, but also the results that can be evaluated during follow-up of patients. Moreover, a method that provides information about the nodule composition, or- ganization and structural characteristics, may orient the kind of therapeutic treatment. Ultrasound, which seems to be a valid support for monitoring laser treatment, is currently used to diagnose nodules and guide the insertion of needles into the thyroid during treatment with conventional B-mode images. New techniques process ultrasonic signals in order to extract further information from the tissue. Elastosonography provides an estimation of tissue stiffness, which seems to be correlated to the pathological condition of the nodule [31-35]. The proposed HyperSPACE algorithm is based on the spectral analysis of the radiofrequency signal that is analyzed in an $\mathrm{N}$ dimensional frequency domain. In this preliminary study, the method was aimed to assess how sets of spectral parameters, called Configuration, occur among benign nodules in different patients and how they change before and after treatment in the same nodule. The interesting clinical relevance, arising from this experimentation, seems to be the correlation between the distributions of Configurations (represented by colors on the B-mode) and extension of necrosis areas induced by laser ablation. The necrosis extension is not accepted as a biunique reliable indicator of the volume nodule reduction in the follow up; however, this result may be important in the perspective of correlating the characteristics identified by HyperSPACE analysis to the response of laser ablation. Indeed, it may identify parameters that foresee the success of laser treatment and may orient the setting of the laser parameters, such as power, energy and duration for a more effective treatment.

The study has highlighted that nodules exhibit different Configurations (colors) distributions that could be indicative of the response of nodular tissue to the laser treatment. This observation may be interpreted as the prevalence of one Configuration (color) in respect of the other or a particular combination of Configurations may be indicative, a priori, of damage that could be induced by laser treatment. In particular, in this pilot study, we have noticed that the prevalence of blue Configuration corresponds to a major necrosis extension; however, a more extensive experiment on a large database is necessary to confirm that.

Moreover, by comparing the HyperSPACE images with those of elastosonography, referring to the same nodules, it can be observed that nodules classified with the same elastographic score or the same echographic appearance have a different Configuration distribution which allowed a classification of nodules into three groups that exhibit different ranges of necrosis extension.

Therefore, HyperSPACE, compared to conventional echographic images and elastosonography seems to provide different and more extensive information about nod- 
ules regarding laser treatment. The decision to set only three Configurations was made in order to simplify the image interpretation by the operator. However, some images show yellow areas obtained from the overlapping of the red and green areas. This could mean that those areas have different characteristics compared to areas characterized by the three well-defined Configurations. The resolution of the characterization of the nodule can be effectively incremented by increasing the number of Configurations, as long as they still offer an easy reading of the images supporting the diagnosis. HyperSPACE images exhibit a high selectivity in detecting nodular areas; instead the elasonography images show colored areas both inside and outside the nodule, making image interpretation and stiffness score evaluation of the nodule more difficult. HyperSPACE was also applied to the nodules 2-3 hours after laser treatment, by using the same Configurations set for characterizing the non-treated nodules. The treated nodules exhibited different distributions of Configurations or the disappearance of some Configurations with respect to the same before ablation and this may mean that the tissue modified its spectral parameters due to damage induced by the laser (data not shown). As said above, in the future, a larger number of patients will be necessary to correlate the Configurations or combinations of them with the success of laser treatment by evaluating not only the necrosis area, but, primarily, the reduction of nodular volume in the follow up of patients.

\section{Conclusion}

The HyperSPACE algorithm could be a valid support to assess the effectiveness of laser ablation and define the nodule characteristics for indicating a priori the success of the treatment but the preliminary results have to be confirmed in a much more extensive study.

Acknowledgments: We wish to thank Elesta SRL of Florence for their technical support and collaboration. We also thank Teresa Rago, MD of the Department of Endocrinology of the University of Pisa for her expertise and precious advice.

\section{References}

1. Papini E, Rago T, Gambelunghe G, et al. Long-term efficacy of ultrasound-guided laser ablation for benign solid thyroid nodules. results of a three-year multicenter prospective randomized trial. J Clin Endocrinol Metab 2014;99:36533659.

2. Papini E, Pacella CM. Percutaneous ethanol injection of benign thyroid nodules and cysts using ultrasound. In: Baskin
HJ (ed). Thyroid Ultrasound and Ultrasound-Guided FNA Biopsy. Boston, MA: Kluwer Academic Publishers, 2000:169-213.

3. Baek JH, Lee JH, Valcavi R, Pacella CM, Rhim H, Na DG.Thermal ablation for benign thyroid nodules: radiofrequency and laser. Korean J Radiol 2011;12:525-540.

4. Kotwal N, Pandit A. Non-invasive and percutaneous ablation of nontoxic solid nodules.Thyroid Res Pract 2014;11:89-93.

5. Achille G, Zizzi S, Di Stasio E, Grammatica A, Grammatica L. Ultrasound-guided percutaneous laser ablation (LA) in treating symptomatic solid benign thyroid nodules: Our experience in 45 patients. Head Neck 2016;38:677-682.

6. Døssing H, Bennedbaek FN, Karstrup S, Hegedüs L. Benign solitary solid cold thyroid nodules: US-guided interstitial laser photocoagulation - initial experience. Radiology 2002;225:53-57.

7. Døssing H, Bennedbaek FN, Hegedüs L. Effect of ultrasound-guided interstitial laser photocoagulation on benign solitary solid cold thyroid nodules a randomised study. Eur J Endocrinol 2005;152:341-345.

8. Gambelunghe G, Bini V, Stefanetti E, et al. Thyroid nodule morphology affects the efficacy of ultrasound-guided interstitial laser ablation: A nested case-control study. Int J Hyperthermia 2014;30:486-489.

9. Gharib H, Hegedüs L, Pacella CM, Baek JH, Papini E. Clinical review: Nonsurgical, image-guided, minimally invasive therapy for thyroid nodules. J Clin Endocrinol Metab 2013;98:3949-3957.

10. Pacella CM, Bizzarri G, Guglielmi R, et al.Thyroid tissue: US-guided percutaneous interstitial laser ablation-a feasibility study. Radiology 2000;217:673-677.

11. Papini E, Pacella CM, Hegedus L. Diagnosis of endocrine disease thyroid ultrasound (US) and US-assisted procedures: from the shadows into an array of applications. Eur J Endocrinol 2014;170:R133-R146.

12. Bandeira-Echtler E, Bergerhoff K, Richter B. Levothyroxine or minimally invasive therapies for benign thyroid nodules. Cochrane Database Syst Rev 2014;(6):CD004098.

13. Papini E, Guglielmi R, Bizzarri G, et al. Treatment of benign cold thyroid nodules: a randomized clinical trial of percutaneous laser ablation versus levothyroxine therapy or follow-up. Thyroid 2007;17:229-235.

14. Haugen BR, Alexander EK, Bible KC, et al. 2015 American Thyroid Association Management Guidelines for Adult Patients with Thyroid Nodules and Differentiated Thyroid Cancer: The American Thyroid Association Guidelines Task Force on Thyroid Nodules and Differentiated Thyroid Cancer. Thyroid 2016;26:1-133.

15. Gharib H, Papini E, Paschke R, et al; AACE/AME/ETA Task Force on Thyroid Nodules. American Association of Clinical Endocrinologists, Associazione Medici Endocrinologi, and European Thyroid Association Medical Guidelines for Clinical Practice for the Diagnosis and Management of Thyroid Nodules. Endocr Pract 2010;16 (Suppl 1):1-43.

16. Benson DM, Rifkin MD, Rose JL, Goldberg BB. Characterization of benign and malignant tissues of the thyroid 
gland. An ultrasonic approach using RF waveform analysis and pattern recognition. Invest Radiol 1983;18:459-462.

17. Wagner RF, Insana MF, Brown DG. Statistical properties of radio-frequency and envelope-detected signals with applications to medical ultrasound. J Opt Soc Am 1987;4:910922.

18. Feleppa EJ, Ketterling JA, Kalisz A, et al. Advanced ultrasonic tissue typing and imaging based on radio-frequency spectrum analysis and neural-network classification for guidance of therapy and biopsy procedures. International Congress Series 2001;1230:346-351.

19. Jurkonis R, Janušauskas A, Marozas V, et al. Algorithms and results of eye tissues differentiation based on RF ultrasound. ScientificWorldJournal 2012;2012:870869.

20. Lizzi FL, Feleppa EJ, Alam SK, Deng CX. Ultrasonic spectrum analysis for tissue evaluation. Pattern Recogn Lett 2003;24:637-658.

21. Lizzi FL, Ostromogilsky M, Feleppa EJ, Rorke MC, Yaremko MM. Relationship of ultrasonic spectral parameters to features of tissue microstructure. IEEE Trans Ultrason Ferroelectr Freq Control 1987;34:319-329.

22. Donohue KD, Huang L, Burks T, Forsberg F, Piccoli CW. Tissue classification with generalized spectrum parameters. Ultrasound Med Biol 2001;27:1505-1514.

23. Masotti L, Biagi E, Granchi S, Breschi L, Magrini E, Di Lorenzo F. Tissue differentiation based on radiofrequency echographic signal local spectral content (RULES: Radiofrequency Ultrasonic Local Estimator). Proc of IEEE U1trasonics Symp 2003;1:1030-1033.

24. Masotti L, Biagi E, Granchi S, et al. Clinical experimentation of FEMMINA and RULES for prostate and breast tumor detection. In: Andre MP. (ed). Acoustical Imaging Berlin, Germany: Springer, 2007;28:349-357.

25. Masotti L, Biagi E, Granchi S, et al. Clinical test of RULES (RULES: radiofrequency ultrasonic local estimators). Proc of IEEE Ultrason Symp 2004;3:2173-2176.

26. Wang J, Kang C, Liu X, et al. Clinical Value of radiofrequency ultrasonic local estimators in classifying breast lesions. J Ultrasound Med 2013;32:83-92.

27. Biagi E, Granchi S, Vannacci E, Lucarini L, Masotti L. Tissue Characterization in Echographic Spectral Hyperspace:
Breast Pathologies Differentiation. Proc of IEEE Ultrason Symp 2010:1388 - 1391.

28. Biagi E, Granchi S, Vannacci E, Masotti L, Martegani A. First Multicenter Experience in Differentiation of Breast Lesions by HyperSPACE SPectral Analysis in Echography. RSNA 2011 Scientific Assembly and Annual Meeting, November 26 - December 2, 2011 ,Chicago IL. http://archive. rsna.org/2011/11009207.html. Accessed January 24, 2017.

29. Granchi S, Vannacci E, Biagi E, Masotti L. Differentiation of Breast Lesions by Use of HyperSPACE: Hyper-Spectral Analysis for Characterization in Echography. Ultrasound Med Biol 2015;41:1967-1980.

30. Granchi S, Vannacci E, Biagi E, Masotti L. Multidimensional spectral analysis of the ultrasonic radiofrequency signal for characterization of media. Ultrasonics 2016;68:89-101.

31. Rago T, Santini F, Scutari M, Pinchera A, Vitti P. Elastography: new developments in ultrasound for predicting malignancy in thyroid nodules. J Clin Endocrinol Metab 2007;92:2917-2922.

32. Hong Y, Liu X, Li Z, Zhang X, Chen M, Luo Z. Real-time ultrasound elastography in the differential diagnosis of benign and malignant thyroid nodules. J Ultrasound Med 2009;28:861-867.

33. Lyshchik A, Higashi T, Asato R, et al. Thyroid gland tumor diagnosis at US elastography. Radiology 2005;237:202211.

34. Rago T, Scutari M, Santini F, et al. Real-Time elastosonography: useful tool for refining the presurgical diagnosis in thyroid nodules with indeterminate or nondiagnostic cytology. J Clin Endocrinol Metab 2010;95:5274-5280.

35. Wang HL, Zhang S, Xin XJ, et al. Application of Real-time Ultrasound Elastography in Diagnosing Benign and Malignant Thyroid Solid Nodules. Cancer Biol Med 2012;9:124127.

36. Scabia M, Biagi E, Masotti L. Hardware and software platform for real-time processing and visualization of echographic radiofrequency signals. IEEE Trans Ultrason Ferroelectr Freq Control 2002;49:1444-1452.

37. Macqueen JB. Some methods for classification and analysis of multivariate observations. Proc Berkeley Symp on Mathematical Statistics and Probability1967;1:281-297. 